\title{
Translationally invariant coupled cluster method in coordinate space for nuclei
}

\author{
I Moliner ${ }^{1}$, N R Walet and R F Bishop \\ Department of Physics, UMIST, PO Box 88, Manchester M60 1QD, UK \\ E-mail: inaki.moliner@uv.es
}

Received 8 February 2002

Published 18 April 2002

Online at stacks.iop.org/JPhysG/28/1209

\begin{abstract}
We study a formulation of the translationally invariant coupled cluster method in coordinate space for finite nuclei. The new formulation remedies convergence problems that plagued previous calculations in configuration space. The method is applied to light nuclei using semi-realistic central interactions.
\end{abstract}

\section{Introduction}

The microscopic description of nuclear systems is complicated by the fact that we need to describe both the short- and long-range correlations caused by the nucleon-nucleon interaction. Several techniques have been used to give an accurate microscopic description of light nuclei, including amongst others variational and Green-function Monte-Carlo methods [1, 2], the Faddeev and Faddeev-Yakubovsky method [3] and the correlated hyperspherical harmonics expansion [4]. The extension of most of these methods to heavier nuclei is far from straightforward, and there are few microscopic methods that have been applied in this new arena. The correlated basis functions method $[5,6]$ has been the most successful and it has been applied to medium-heavy closed-shell nuclei $\left({ }^{16} \mathrm{O},{ }^{40} \mathrm{Ca}, \ldots\right)$. In this paper we are going to use a method inspired by the coupled cluster method (CCM).

The CCM has been used extensively to describe many-body systems in many areas of physics, including nuclear physics, the field in which it was first introduced [7, 8]. The configuration-space coupled cluster method $[9,10]$ has recently been applied to ${ }^{16} \mathrm{O}[11]$. However, it is worth mentioning at this point that our method, although akin to the standard coupled cluster techniques, differs greatly from those used in [11], mainly in two aspects: the preservation of translational invariance and the formulation in coordinate space, rather than in configuration space. Thus these very different calculations cannot be directly compared.

When dealing with finite systems such as nuclei it is very important to treat the centreof-mass motion correctly. This was the main motivation for the introduction of a version

1 Present address: Departamento de Física Atómica y Nuclear, Universidad de Valencia, Dr Moliner 50, 46100 Burjassot, Spain. 
of the CCM that incorporates the required translational invariance from the very outset, the translationally invariant coupled cluster method (TICCM) [12]. This method has been implemented at the so-called SUB(2) level of approximation, in which only one- and twobody terms are considered in the cluster excitation operator. This so-called TICC2 scheme was initially applied to the ${ }^{4} \mathrm{He}$ nucleus. The configuration-space version suffers from poor convergence, even for soft semi-realistic nucleon-nucleon interactions [12], and it was realized that a linearized version of the method can easily be formulated in coordinate space. This method has been applied to various nuclei in the 0p-shell $[13,14]$. The results obtained with this linearized wavefunction and state-dependent correlations are good for semi-realistic central forces. However, once realistic nuclear interactions, which have a strongly repulsive core, are used, the overall quality of the results deteriorates.

In order to tackle this problem more correlations must be included in the wavefunction, and there are various ways to do it. We have used in the past a mixed scheme including Jastrow correlations, which was first applied to ${ }^{4} \mathrm{He}[14]$ and has recently been extended to heavier nuclei [15]. The use of Jastrow correlations complicates the calculations considerably, but the mixture of short-range Jastrow scalar correlations and medium- and long-range TICI2 state-dependent correlations gives good results. Another way of including more correlations is, of course, to go beyond the linear TICI2 approximation. It is therefore interesting to ask whether the inclusion of the higher order correlation terms present in the TICCM might be able to give similarly improved results without the complicated Jastrow factors. In this work we focus on the quadratic terms of the TICC 2 wavefunction. In the original study of ${ }^{4} \mathrm{He}$ [12] these terms were shown to be almost negligible, but this does not prohibit larger effects for heavier systems, and we have recently investigated their inclusion for general bosonic systems [16] in configuration space. The additional terms turn out to give a quite significant contribution to the ground-state energy for systems with boson number greater than 4 . At the same time, calculations for bosonic systems in configuration space have proved to be quite time-consuming, and present convergence problems as mentioned above. This problem disappears when we reformulate the TICCM in coordinate space [17]. The question thus arises whether this also holds for finite nuclei. To this end the methodology is extended from bosonic to fermionic systems, and the resulting TICC2 wavefunction is used to study closed-shell nuclei within the 0p-shell, using semi-realistic central forces with a V4 structure (scalar plus spin-, isospin- and space-exchange terms). The wavefunction we are presently using is not sophisticated enough to provide a fully realistic account of nuclear properties, which would also require the use of high-quality realistic two-body interactions, such as the Argonne $v_{18}$ interaction [18] (plus three-body forces). Our main objective in this work is to test the relevance of the second-order terms in the TICC 2 wavefunction and implement the method for finite nuclei; to attain this goal semi-realistic interactions are the best choice, since they are simple, yet capable of a qualitative description of nuclei.

In the following section we discuss the TICC 2 wavefunction we employ, and in section 3 the equations and diagrammatical expansions involved are explained. Section 4 is devoted to the results obtained and conclusions are drawn in section 5.

\section{The TICC2 wavefunction}

The general CCM ansatz for the ground state wavefunction of an $A$-particle system is of the exponential form

$$
|\Psi\rangle=\exp (S)|\Phi\rangle,
$$


where $S$ is a sum of one-body, two-body, etc, operators acting on an uncorrelated reference state $|\Phi\rangle$ with the right symmetry. Unless both the reference state and the excitation operators are properly constructed to be translationally invariant, the centre of mass is not correctly treated. When the operator $S$ is restricted to a sum of one- and two-body excitations one can build a translationally invariant wavefunction by using an appropriate admixture of the oneand two-body terms, provided that the centre-of-mass contribution can be factorized exactly in the reference state. This is the main motivation to choose harmonic oscillator (HO) states for the uncorrelated $A$-particle reference state. For a bosonic system the correlated wavefunction was shown to be of the form [12]

$$
|\Psi\rangle=: \exp \left(S^{(1,2)}\right):|\Phi\rangle,
$$

where the reference state is

$$
|\Phi\rangle=\frac{1}{\sqrt{A !}}\left(a_{000}^{\dagger}\right)^{A}|0\rangle
$$

and $|0\rangle$ is the vacuum state. The operators $a_{n l m}^{\dagger}$ create the single-particle harmonic oscillator states, $a_{n l m}^{\dagger}|0\rangle=|n l m\rangle$, in a harmonic potential well with natural frequency $\omega$. The translationally invariant $S^{(1,2)}$ operator is given by

$$
S^{(1,2)}=\sum_{n=1}^{\infty} \mathcal{S}(n) \sum_{n_{1} n_{2} l_{1}}\left\langle n 000 ; 0 \mid n_{1} l_{1} n_{2} l_{1}\right\rangle\left[a_{n_{1} l_{1}}^{\dagger} \times a_{n_{2} l_{1}}^{\dagger}\right]_{0}^{0} a_{000}^{2} .
$$

The action of this operator promotes a pair of particles from the harmonic oscillator reference state, ensuring that the centre of mass of the pair is not excited by imposing a suitable coupling via the Brody-Moshinsky brackets in equation (4). The angular momenta of the single-particle states are coupled to zero total angular momentum to ensure rotational invariance. The normal ordering in equation (2) is needed because when the operator $S^{(1,2)}$ acts more than once noninvariant terms are produced, which can be eliminated using the normal-order prescription. The operator $S^{(1,2)}$ mixes one- and two-particle excitations, because one or the other (but not both) of the indices $n_{1}$ and $n_{2}$ can be null. Even though the translationally invariant wavefunction (2) is constructed in configuration space, it can be most easily represented in coordinate space in terms of a two-body function $f\left(r_{i j}\right)$, depending only on the relative coordinate of a pair. Thus the TICC 2 wavefunction can be expressed in coordinate space as $[19,20]$

$$
\begin{aligned}
\Psi\left(\mathbf{r}_{1}, \ldots, \mathbf{r}_{A}\right) & =\left(1+\sum_{i<j} f\left(r_{i j}\right)+\frac{1}{2 !} \sum_{i<j} \sum_{k<l}^{\prime} f\left(r_{i j}\right) f\left(r_{k l}\right)\right. \\
& \left.+\frac{1}{3 !} \sum_{i<j} \sum_{k<l}^{\prime} \sum_{m<n}^{\prime \prime} f\left(r_{i j}\right) f\left(r_{k l}\right) f\left(r_{m n}\right)+\cdots\right) \Phi\left(\mathbf{r}_{1}, \ldots, \mathbf{r}_{A}\right),
\end{aligned}
$$

where $\Phi\left(\mathbf{r}_{1}, \ldots, \mathbf{r}_{A}\right)$ is the uncorrelated $\mathrm{HO}$ wavefunction. The primed sums denote that all the indices in the nested sums are distinct, showing explicitly the characteristic CCM property of dealing with independent excitations only. The above wavefunction also corresponds to the frequently used independent pair approximation [21] for Jastrow correlated wavefunctions, where the Jastrow correlation is parameterized in the form $\left(1+f\left(r_{i j}\right)\right)$. If we only keep up to linear terms in $f(r)$ in the wavefunction (5), we obtain the wavefunction used in the translationally invariant configuration-interaction (TICI2) method. The problem is thus reduced to the determination of the pair correlation function $f(r)$. The intermediate 
normalization condition $\langle\Phi \mid \Psi\rangle=1$, which is implicit in the CCM, implies that the correlation function must be restricted by the relation

$$
\left\langle\Phi\left|\left(\sum_{i<j} f\left(r_{i j}\right)+\frac{1}{2 !} \sum_{i<j} \sum_{k<l}^{\prime} f\left(r_{i j}\right) f\left(r_{k l}\right)+\cdots\right)\right| \Phi\right\rangle=0
$$

to ensure that the correlations are orthogonal to the reference state.

Wavefunction (5) has been used for bosonic systems [17], where it was realized that the relevant contributions to the ground-state energies were mainly given by the first- and second-order terms, with the higher orders (up to fourth-order terms are allowed for bosons) giving negligible contributions. Although wavefunction (5) was derived for bosonic systems, there is nothing that prevents us from using it for fermions, provided that the starting reference state has the appropriate symmetries. Fermionic systems are more complicated than bosonic systems due to the Pauli principle and the necessity of considering different space states, so we have restricted the wavefunction and considered only up to quadratic terms, which are relevant in the bosonic case, as mentioned above. We shall refer to this second-order truncated TICC2 wavefunction as TICC2/2 (similarly, the linear truncation, TICI2, can also be referred to as TICC2/1). The TICC2/2 wavefunction is thus

$$
|\Psi\rangle=\left(1+\sum_{i<j} f\left(r_{i j}\right)+\frac{1}{2 !} \sum_{i<j} \sum_{k<l}^{\prime} f\left(r_{i j}\right) f\left(r_{k l}\right)\right)|\Phi\rangle,
$$

where the reference state, $|\Phi\rangle$, is now a single Slater determinant of the lowest filled HO states, appropriate for spin and isospin saturated systems, and which maintains the property of factorizing the centre-of-mass contribution. We then attempt to obtain approximate solutions to the Schrödinger equation, using techniques common to CCM.

\section{Equations and diagrams}

Once we have defined the correlated wavefunction, the typical CCM procedure allows one to find equations for the ground-state energy and the coefficients in the wavefunction by projecting the Schrödinger equation onto the reference state and all the other states that are present in the $S$ operator used to build the wavefunction. In fact, the traditional CCM way is first to premultiply the Schrödinger equation by $\mathrm{e}^{-S}$ to take advantage of the linked cluster expansion of the $\mathrm{e}^{-S} H \mathrm{e}^{S}$ operator [8]. This is not feasible in our case because we are working within the coordinate-space representation, and even if we were using the configuration-space representation, equation (2), the use of the normal ordering prevents us from using the linked cluster expansion. Hence the projection will be performed directly onto the Schrödinger equation, without any previous manipulation. If we project onto the reference state and onto a generic excitation with the same characteristics as $f$, and which is described by multiplying $|\Phi\rangle$ with a function $g$, we get

$$
\begin{aligned}
& \langle\Phi|H| \Psi\rangle=E, \\
& \langle\Phi|g H| \Psi\rangle=E\langle\Phi|g| \Psi\rangle,
\end{aligned}
$$

where the non-relativistic Hamiltonian $H=T+V$ is the sum of the kinetic energy operator and a two-body nucleon-nucleon interaction,

$$
H=-\frac{\hbar}{2 m} \sum_{i} \nabla_{i}^{2}+\sum_{i<j} V_{i j}
$$




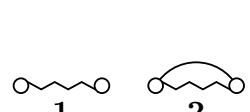

1

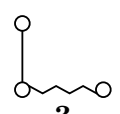

3

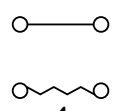

4

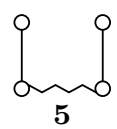

5

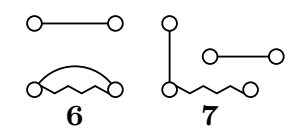

7

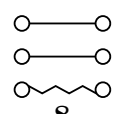

8

Figure 1. Norm diagrams in the $G$ equation. These diagrams correspond to the calculation of $\langle\Phi|G| \Psi\rangle$. Note that diagrams 4 and 6-8 have disconnected correlation functions. The maximum number of particles involved is 6 .

Equations (8) and (9) provide us with either an integro-differential equation for arbitrary $g$ and $f$, or a set of nonlinear coupled equations if we expand $g$ and $f$ using a suitable basis (Gaussians, for example). We would like to have a fully arbitrary $g$, in which case the vanishing of an integral linear in $g$ would imply the vanishing of the integrand. However, $g$ also satisfies the intermediate normalization condition $\langle\Phi|g| \Phi\rangle=0$. Nevertheless, a simple way to get manageable equations is to express $g$ in terms of a fully arbitrary function as $g=G-\langle\Phi|G| \Phi\rangle$, which obviously satisfies $\langle\Phi|g| \Phi\rangle=0$. By doing so, equation (9) becomes

$$
\langle\Phi|G H| \Psi\rangle-\langle\Phi|G| \Phi\rangle\langle\Phi|H| \Psi\rangle=E\langle\Phi|G| \Psi\rangle-E\langle\Phi|G| \Phi\rangle\langle\Phi \mid \Psi\rangle,
$$

which, using the equation $\langle\Phi|H| \Psi\rangle=E$ and the intermediate normalization, $\langle\Phi \mid \Psi\rangle=1$, results in

$$
\langle\Phi|G H| \Psi\rangle=E\langle\Phi|G| \Psi\rangle .
$$

This looks exactly like the equation we started with, but the difference is hidden, because now $\langle\Phi|G| \Phi\rangle \neq 0$.

We can expand the previous expressions in a diagrammatic fashion where circles represent particles, solid lines represent correlation functions $f$, wavy lines represent $G$, dashed lines stand for the interaction, and crosses indicate the particle the kinetic energy operator is acting on. To further clarify the meaning of the diagrammatic notation consider the following diagram and its explicit meaning:

$$
\stackrel{-}{-} \equiv\left\langle\Phi\left|G^{*}\left(r_{12}\right) V\left(r_{34}\right) f\left(r_{13}\right) f\left(r_{24}\right)\right| \Phi\right\rangle
$$

The labelling of the particles in the diagram is irrelevant, since the result is invariant under permutations, and the statistical weight which accompanies every diagram accounts for the sum over all permutations. The equation for the energy then reads

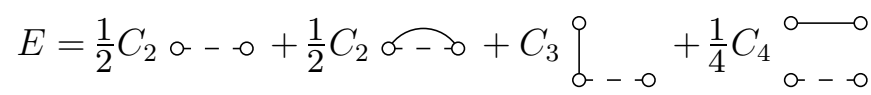

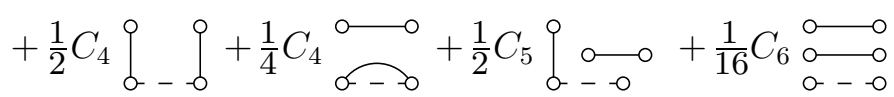

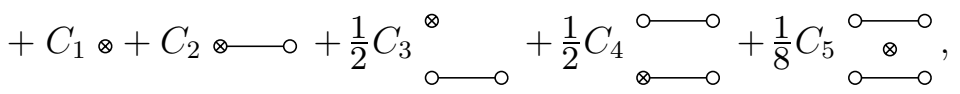

where $C_{k} \equiv A(A-1) \ldots(A-k+1)$. We have a similar expression for the $G$ equation, the projection onto $\langle\Phi| G$, but involving many more diagrams. The diagrams corresponding to the norm, kinetic and potential parts $(\langle\Phi|G| \Psi\rangle,\langle\Phi|G T| \Psi\rangle$ and $\langle\Phi|G V| \Psi\rangle$ respectively) in this equation are displayed in figures $1-3$. The statistical weights associated with the diagrams are shown in table 1 , and include the additional $1 / 2$ ! factor in the quadratic diagrams coming from wavefunction (7). It is in the diagrammatic expansion where a significant difference between the bosonic and fermionic cases arises. In the bosonic case the unlinked diagrams factorize because in the reference state all the particles are in the lowest HO state $(n l m)=(000)$, and 


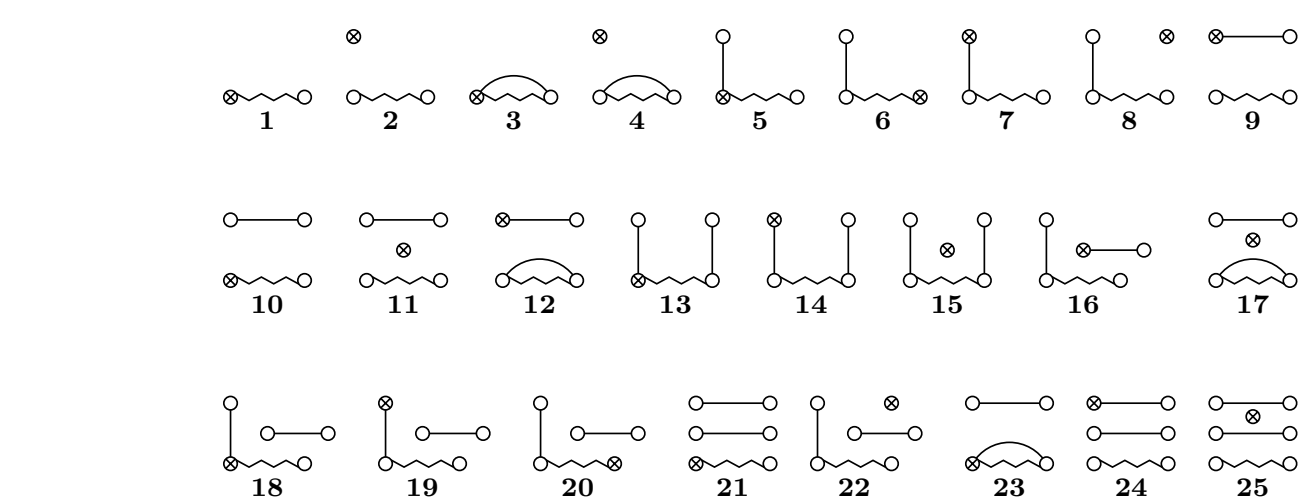

Figure 2. Kinetic energy diagrams in the $G$ equation. The kinetic energy diagrams correspond to $\langle\Phi|G T| \Psi\rangle$. Diagrams 10, 11 and 17-25 have disconnected correlation functions. The maximum number of particles involved is 7 (diagram 25).

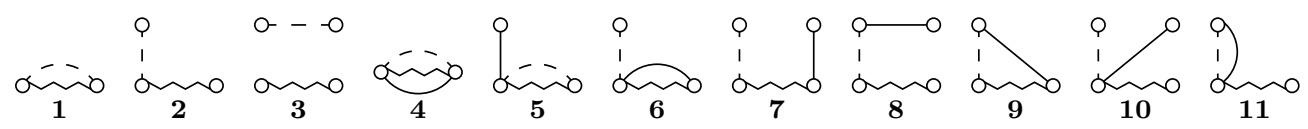

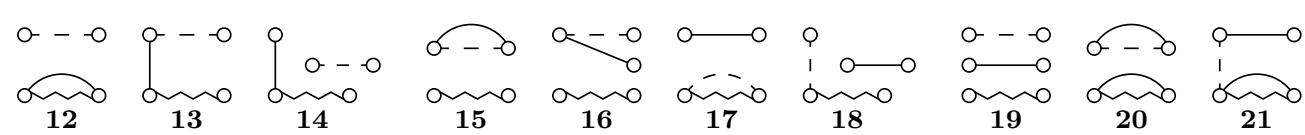

$$
\begin{aligned}
& \underbrace{--0}_{22}
\end{aligned}
$$

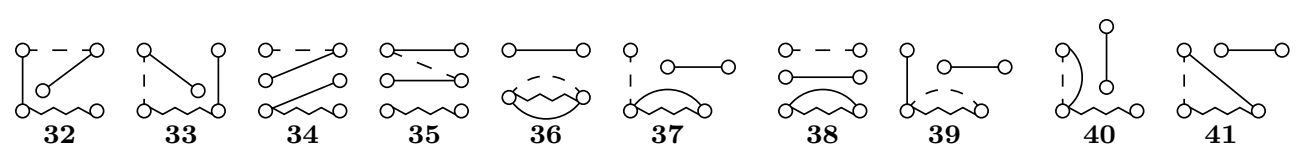

$$
\begin{aligned}
& \underbrace{--}_{42} \underbrace{-}_{43}
\end{aligned}
$$

Figure 3. Diagrams involving the potential in the $G$ equation, corresponding to $\langle\Phi|G V| \Psi\rangle$. Diagrams $17-19$ and 36-51 have disconnected correlation functions. The maximum number of particles involved is 8 (diagram 51), and there are three seven-particle diagrams (45, 48 and 49).

thus a contribution such as $\left\langle\Phi\left|V\left(r_{12}\right) f\left(r_{34}\right)\right| \Phi\right\rangle$ evaluates to $\left\langle\Phi\left|V\left(r_{12}\right)\right| \Phi\right\rangle \times\left\langle\Phi\left|f\left(r_{34}\right)\right| \Phi\right\rangle$ and, since $\left\langle\Phi\left|f\left(r_{i j}\right)\right| \Phi\right\rangle=0$, all the diagrams containing a disconnected $f$ give a null contribution. This means that, even without truncating the wavefunction, the equations are of finite order because there is an upper limit to the number of linked $f$ correlations that can appear in a given diagram (four with two-body interactions: two linked by $G$ and two by the interaction). But this is not the case with fermions because the permutations in the reference state involve different $\mathrm{HO}$ states. Now we are not able to compute $\left\langle\Phi\left|V\left(r_{12}\right) f\left(r_{34}\right)\right| \Phi\right\rangle$ as 
Table 1. Statistical weights for the different norm $(N)$, kinetic $(T)$ and potential $(V)$ diagrams in the $G$ equation (including the $1 / 2$ ! factor coming from the wavefunction in the quadratic diagrams). $C_{k} \equiv A(A-1) \ldots(A-k+1)$, where $k$ also indicates the number of particles involved in the diagram.

\begin{tabular}{lllllllllll}
\hline$N_{1} \ldots N_{8}$ & $\frac{1}{2} C_{2}$ & $\frac{1}{2} C_{2}$ & $C_{3}$ & $\frac{1}{4} C_{4}$ & $\frac{1}{2} C_{4}$ & $\frac{1}{4} C_{4}$ & $\frac{1}{2} C_{5}$ & $\frac{1}{16} C_{6}$ & & \\
$T_{1} \ldots T_{10}$ & $C_{2}$ & $\frac{1}{2} C_{3}$ & $C_{2}$ & $\frac{1}{2} C_{3}$ & $C_{3}$ & $C_{3}$ & $C_{3}$ & $C_{4}$ & $\frac{1}{2} C_{4}$ & $\frac{1}{2} C_{4}$ \\
$T_{11} \ldots T_{20}$ & $\frac{1}{4} C_{5}$ & $\frac{1}{2} C_{4}$ & $C_{4}$ & $C_{4}$ & $\frac{1}{2} C_{5}$ & $C_{5}$ & $\frac{1}{2} C_{4}$ & $\frac{1}{4} C_{5}$ & $\frac{1}{2} C_{5}$ & $\frac{1}{2} C_{5}$ \\
$T_{21} \ldots T_{25}$ & $\frac{1}{2} C_{5}$ & $\frac{1}{2} C_{6}$ & $\frac{1}{8} C_{6}$ & $\frac{1}{4} C_{6}$ & $\frac{1}{16} C_{7}$ & & & & & \\
$V_{1} \ldots V_{10}$ & $\frac{1}{2} C_{2}$ & $C_{3}$ & $\frac{1}{4} C_{4}$ & $\frac{1}{2} C_{2}$ & $C_{3}$ & $C_{3}$ & $C_{4}$ & $C_{4}$ & $C_{3}$ & $C_{4}$ \\
$V_{11} \ldots V_{20}$ & $C_{3}$ & $\frac{1}{4} C_{4}$ & $C_{4}$ & $\frac{1}{2} C_{5}$ & $\frac{1}{4} C_{4}$ & $\frac{1}{2} C_{5}$ & $\frac{1}{4} C_{4}$ & $\frac{1}{2} C_{5}$ & $\frac{1}{8} C_{6}$ & $\frac{1}{4} C_{4}$ \\
$V_{21} \ldots V_{30}$ & $C_{4}$ & $\frac{1}{2} C_{5}$ & $\frac{1}{2} C_{4}$ & $C_{4}$ & $\frac{1}{2} C_{4}$ & $C_{4}$ & $C_{5}$ & $C_{5}$ & $\frac{1}{4} C_{6}$ & $\frac{1}{2} C_{5}$ \\
$V_{31} \ldots V_{40}$ & $C_{5}$ & $C_{5}$ & $C_{5}$ & $C_{6}$ & $\frac{1}{4} C_{6}$ & $\frac{1}{4} C_{4}$ & $\frac{1}{2} C_{5}$ & $\frac{1}{8} C_{6}$ & $\frac{1}{2} C_{5}$ & $\frac{1}{2} C_{5}$ \\
$V_{41} \ldots V_{50}$ & $\frac{1}{2} C_{5}$ & $\frac{1}{2} C_{6}$ & $\frac{1}{2} C_{6}$ & $\frac{1}{2} C_{6}$ & $\frac{1}{16} C_{6}$ & $\frac{1}{4} C_{7}$ & $\frac{1}{8} C_{6}$ & $\frac{1}{8} C_{7}$ & $\frac{1}{4} C_{7}$ & $\frac{1}{2} C_{6}$ \\
$V_{51}$ & $\frac{1}{32} C_{8}$ & & & & & & & & & \\
\hline
\end{tabular}

$\left\langle\Phi\left|V\left(r_{12}\right)\right| \Phi\right\rangle \times\left\langle\Phi\left|f\left(r_{34}\right)\right| \Phi\right\rangle$, and therefore such contributions are not null anymore. Thus if the wavefunction is not truncated, the number of diagrams to consider is, in principle, infinite. Even when the wavefunction is truncated (to second order, in the present case) the number of diagrams to consider is much greater than in the bosonic case due to the inclusion of contributions with disconnected correlation functions. Nevertheless, one can wonder about the relevance of such diagrams in the fermionic case, and whether the fact that they vanish identically in a bosonic system will be reflected in the results for fermions. We will therefore check the importance of these diagrams by computing them separately.

The numerical calculation is performed using a Gaussian expansion, which provides an excellent representation of the correlation function when using both positive and negative Gaussian parameters in combination with the harmonic oscillator single-particle states. The actual size of the Gaussian set depends on the nature of the interaction, but usually a small number (10-14) is enough to reach convergence. If the radial part of the nucleonnucleon interaction has a Gaussian form too (as is the case for those we will use), all the integrals appearing in the calculation can be computed analytically using recurrence relations for Gaussian integrals with polynomial parts. Expanding both $G$ and $f$ in the above-mentioned Gaussian basis, we get a system of nonlinear coupled equations for the coefficients of the Gaussian expansion of $f$. Since the wavefunction is quadratic in $f$, so is the system of equations. A problem is to pick the physical solution from the many solutions such nonlinear equations can possess. This can be overcome by using some suitable procedure. In the present case we have used the PITCON continuation routine [22], which computes a sequence of solution points along a one-dimensional manifold of a parametric system of nonlinear equations. We start from the known solution of the linear equations, and we follow the development of the full solution as the nonlinear part of the equations is slowly switched on.

The reference state $|\Phi\rangle$ is chosen as a Slater determinant of harmonic oscillator singleparticle wavefunctions in a Cartesian basis, and we have chosen non-spherical reference states for ${ }^{8} \mathrm{Be}\left(|\Phi\rangle=\left|(0,0,0)^{4}(0,0,1)^{4}\right\rangle\right.$ and ${ }^{12} \mathrm{C}\left(|\Phi\rangle=\left|(0,0,0)^{4}(1,0,0)^{4}(0,1,0)^{4}\right\rangle\right.$ in order to have spin and isospin saturation, within an $L S$ coupling scheme. Thus, the rotation symmetry is broken in these cases and we do not get a strict estimate for the ground-state energy, but for a weighted average of the $J=0,2,4$ states. 
Table 2. Binding energies (in $\mathrm{MeV}$ ) for spin and isospin saturated nuclei in the $0 \mathrm{p}$-shell, using the $\mathrm{B} 1$ and MS3 interactions. $\alpha$ is the HO inverse length parameter (in $\mathrm{fm}^{-1}$ ), TICC2/1 and TICC2/2 stand for linear or linear-plus-quadratic wavefunctions, as explained in the text, and $*$ means that unlinked diagrams are not considered. The eight-particle unlinked diagram 51 in figure 3 is not considered for any of the nuclei, and diagrams 45,48 and 49 are excluded in the ${ }^{16} \mathrm{O}$ calculations.

\begin{tabular}{ccccccc}
\hline & & $\alpha$ & TICC2/1* & TICC2/1 & TICC2/2* & TICC2/2 \\
\hline \multirow{4}{*}{ B1 } & ${ }^{4} \mathrm{He}$ & 0.729 & 37.86 & 37.86 & 37.92 & 37.92 \\
& ${ }^{8} \mathrm{Be}$ & 0.595 & 48.96 & 49.18 & 50.04 & 49.96 \\
& ${ }^{12} \mathrm{C}$ & 0.595 & 84.95 & 84.91 & 86.16 & 86.17 \\
& ${ }^{16} \mathrm{O}$ & 0.602 & 146.60 & 145.92 & 149.36 & 149.27 \\
& ${ }^{4} \mathrm{He}$ & 0.713 & 25.41 & 25.41 & 25.59 & 25.59 \\
$\mathrm{MS} 3$ & ${ }^{8} \mathrm{Be}$ & 0.582 & 25.84 & 26.26 & 28.37 & 28.35 \\
& ${ }^{12} \mathrm{C}$ & 0.588 & 46.06 & 46.22 & 50.53 & 50.52 \\
& ${ }^{16} \mathrm{O}$ & 0.596 & 85.94 & 85.56 & 94.71 & 94.81 \\
\hline
\end{tabular}

\section{Results}

The energies obtained for spin and isospin saturated nuclei in the $0 \mathrm{p}$-shell are shown in table 2. The nucleon-nucleon interactions used are the Gaussian Brink-Boeker B1 [23] and modified Afnan-Tang MS3 [24] central potentials (the MS3 is the Afnan-Tang S3 interaction [25] supplemented in the odd channels with the repulsive part of the even ones). The values of the HO inverse length parameter are taken from [13], in order to be able to compare the results for linear correlations with those presented there. These values of the HO parameter are those that optimize the energy values for the linear wavefunction and we shall investigate the $\alpha$-dependence of the quadratic results later. A full calculation with the quadratic wavefunction (7) would require the computation of an eight-particle diagram. This is an extremely involved computation (compared to a six-particle diagram, the evaluation of an eight-particle diagram requires a computer time roughly 200 times greater in ${ }^{8} \mathrm{Be}$, and 900 times greater in ${ }^{16} \mathrm{O}$ ), although a seven-particle calculation is feasible in the case of ${ }^{8} \mathrm{Be}$ and ${ }^{12} \mathrm{C}$. To see the effect of the unlinked diagrams (the disconnected ones giving null contributions in the bosonic case) their contribution is computed separately. It is worth noting that if we do not consider the disconnected diagrams, the maximum number of particles to deal with is six, and all these diagrams are included in our calculation. The linear TICC2/1 results (those obtained considering all the diagrams in the calculation, both linked and unlinked) coincide precisely with those in [13]. Even though we can mathematically show the equivalence of these two approaches, it is again worth noting that the procedure is completely different from that used in [13], where the linear form of the wavefunction was used to compute the expectation value of the energy variationally, by solving a generalized eigenvalue problem for the coefficients of the Gaussian expansion.

First we comment on the role of the unlinked diagrams. Their contribution is small in the linear case, although this depends strongly on the system and interaction considered (except in the ${ }^{4} \mathrm{He}$ case, of course, where these diagrams do not contribute), ranging from 0.04 to $0.68 \mathrm{MeV}$ with the B1 interaction, and from 0.16 to $0.42 \mathrm{MeV}$ with the MS3 potential. In a sense, these diagrams give us a 'fine tuning' of the wavefunction, because although their relevance for the value of the ground-state energy is small, we can see that they are important, for instance, for the fulfilment of the intermediate orthogonality condition. This is an interesting check of the quality of the approximations, and we can compute the 
Table 3. Comparison of binding energies (in $\mathrm{MeV}$ ) for ${ }^{8} \mathrm{Be}$ and ${ }^{12} \mathrm{C}$ using the MS3 interaction when different sets of 'unlinked' diagrams are added to the linked ones. The values of $\langle\Phi \mid \Psi\rangle-1$ are also quoted to check the fulfilment of the intermediate normalization condition. TICC $2 / 1^{*}$ and TICC $2 / 2^{*}$ stand for the linear and linear-plus-quadratic results without the unlinked diagrams, $u^{1}$ for the unlinked linear diagrams and $u^{2}$ for the unlinked quadratic diagrams (excluding diagram 51 in figure 3 ).

\begin{tabular}{llllll}
\hline & \multicolumn{2}{c}{${ }^{8} \mathrm{Be}$} & & \multicolumn{2}{c}{${ }^{12} \mathrm{C}$} \\
\cline { 2 - 3 } \cline { 5 - 6 } \cline { 5 - 6 } & $E$ & $\langle\Phi \mid \Psi\rangle-1$ & & $E$ & $\langle\Phi \mid \Psi\rangle-1$ \\
\hline $\mathrm{TICC} 2 / 1^{*}$ & 25.84 & $5 \times 10^{-4}$ & & 46.06 & $2 \times 10^{-5}$ \\
$\mathrm{TICC} 2 / 1^{*}+u^{1}$ & 26.26 & $2 \times 10^{-14}$ & & 46.22 & $2 \times 10^{-13}$ \\
$\mathrm{TICC} 2 / 2^{*}$ & 28.37 & $2 \times 10^{-3}$ & & 50.53 & $2 \times 10^{-4}$ \\
$\mathrm{TICC} 2 / 2^{*}+u^{1}$ & 28.61 & $1 \times 10^{-3}$ & & 50.58 & $5 \times 10^{-5}$ \\
TICC2 $/ 2^{*}+u^{1}+u^{2}$ & 28.35 & $1 \times 10^{-6}$ & & 50.52 & $1 \times 10^{-6}$ \\
\hline
\end{tabular}

expectation value of the correlation function, $\left\langle\Phi\left|f_{12}\right| \Phi\right\rangle$ in the linear case or $\langle\Phi| f_{12}+\frac{1}{4}(A-2)$ $(A-3) f_{12} f_{34}|\Phi\rangle$ in the quadratic one, which should be null due to the intermediate normalization condition. In table 3 we show the results obtained considering different sets of diagrams, and the expectation value of the correlation function is quoted alongside the energies. We note that when including the unlinked diagrams, although the value of the ground-state energy does not change much (especially in the quadratic case), the expectation value of the correlation function decreases by several orders of magnitude, rapidly approaching zero. The implication is that when not considering the whole set of diagrams we are breaking (although only slightly) the orthogonality condition, and the excited states are not exactly orthogonal to the reference state.

The quadratic computation, unlike its linear counterpart, is not complete, because we here disregard the final unlinked eight-particle diagram (in the ${ }^{16} \mathrm{O}$ case also the seven-particle ones), but the contribution to the energy of the unlinked diagrams is smaller than that in the linear case, ranging between $0.01 \mathrm{MeV}$ and $0.10 \mathrm{MeV}$. The inclusion of the second-order diagrams in the calculation of $f$ makes the unlinked diagrams less important, even if the number of unlinked diagrams in the quadratic case is much higher than that in the linear case. This fact is very important for the future extension of the computations to more complicated correlations, because this indicates that the relevant set of diagrams with the quadratic wavefunction consist of linked diagrams only, with the unlinked contributions almost cancelling out.

The actual contribution of the quadratic terms to the ground-state energies obviously depends on the interaction considered. The quadratic results are not optimized with respect to the harmonic oscillator parameter value, but are computed for the values that optimized the linear energy. It is not obvious that the optimal HO parameter values should be the same; we will address this issue later. For the current choice, the increase in binding energy is, roughly, around $2 \%$ for B 1 , and around $10 \%$ for MS3. The shape of the TICC $2 / 2$ correlation functions is displayed in figure 4, for the four nuclei and two interactions considered. As expected, the shape of the correlation function follows the shape of the interaction, and in this way the B1 correlation is smoother than the MS3 one, since the latter interaction has a markedly stronger core. In both cases the correlations for the different nuclei show a definite trend to smaller cusps and a smoother asymptotic behaviour as we increase the mass of the nucleus, with the exception of the case of ${ }^{8} \mathrm{Be}$, probably due to the difficulty of describing this strongly deformed nucleus in our spherical model. We can also compare the correlation functions for the linear TICC2/ 1 and quadratic TICC $2 / 2$ cases, as is shown for ${ }^{16} \mathrm{O}$ in figure 5 . The effect 


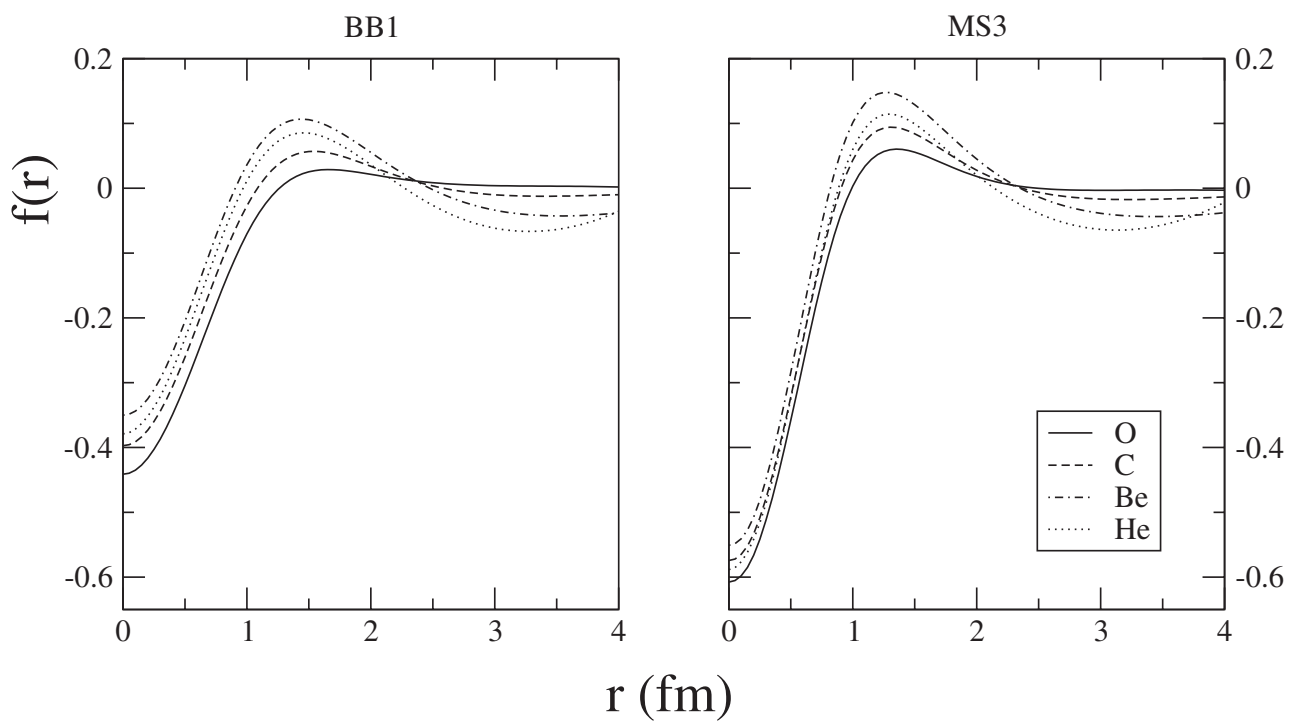

Figure 4. Correlation functions obtained in the TICC2/2 calculations with the Brink-Boeker B1 interaction and modified Afnan-Tang MS3 interaction. The values of $\alpha$ are given in table 2.

BB1

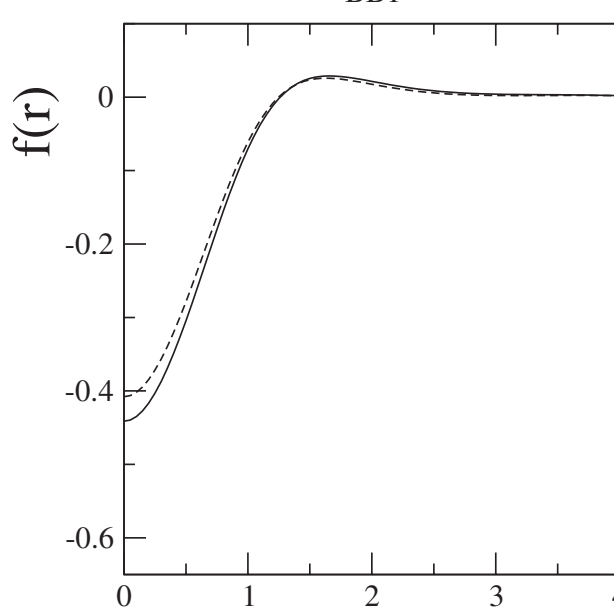

MS3

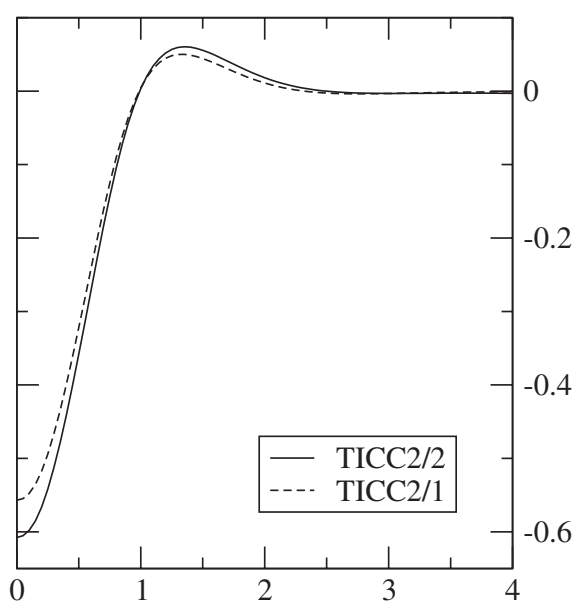

$\mathrm{r}(\mathrm{fm})$

Figure 5. Correlation functions in linear TICC $2 / 1$ and quadratic TICC $2 / 2$ truncations for the Brink-Boeker B1 and modified Afnan-Tang MS3 interactions for ${ }^{16} \mathrm{O}$. The values of $\alpha$ are quoted in table 2.

of including more correlations, as in the quadratic case, is to deepen the correlation at the origin, which is related to the repulsive core, and slightly sharpen the cusp around 1.2-1.5 fm, located at the minimum of the Wigner component of the interactions.

We have noted before that the energies for the TICC $2 / 2$ wavefunction were not optimized with respect to the $\mathrm{HO}$ parameter, but merely calculated at the same points that minimize the linear results. The ${ }^{8} \mathrm{Be}$ nucleus is a simple enough system to perform a variational search including all the diagrams, but for the heavier nuclei we have used only the linked diagrams. 


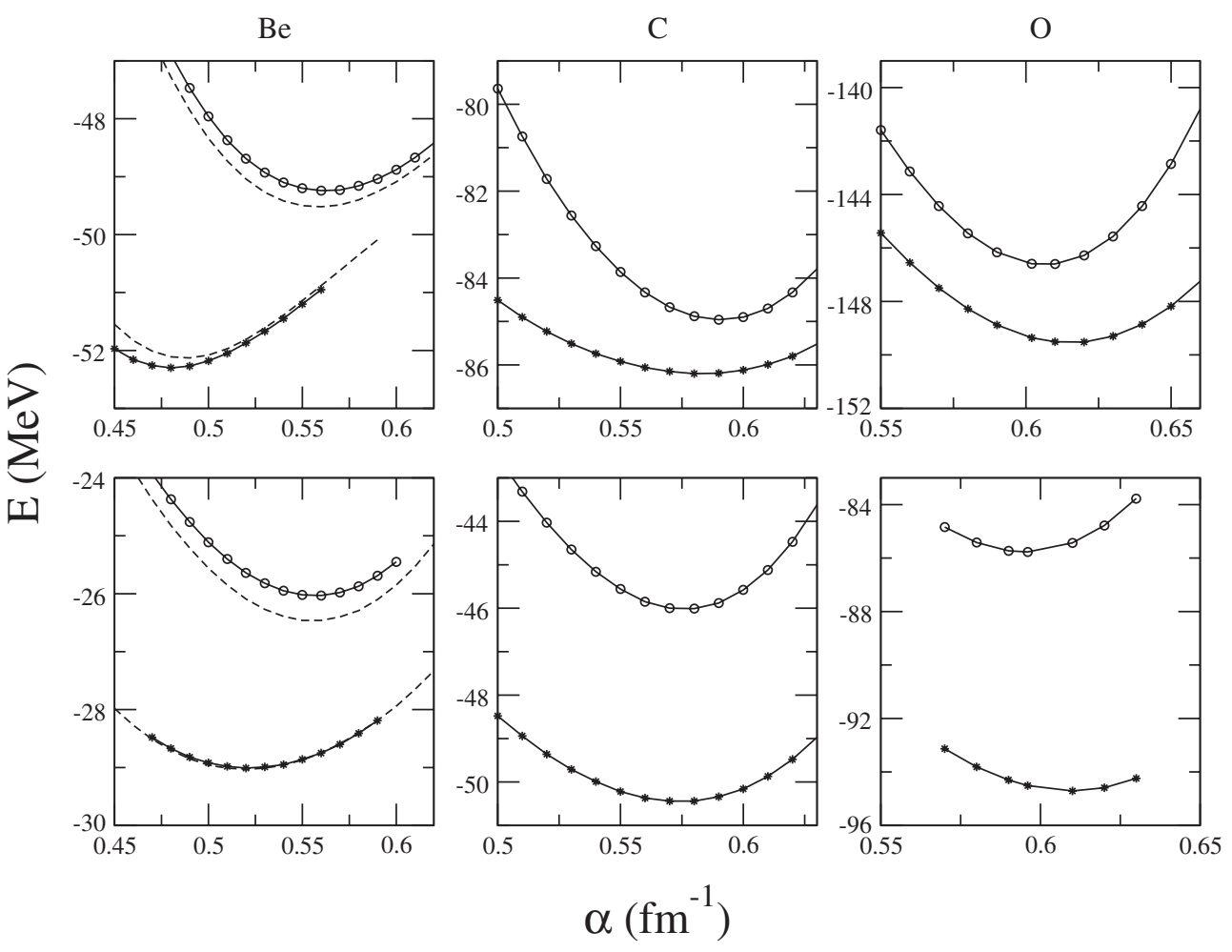

Figure 6. Variation of the energy with respect to the value of the HO inverse length parameter, $\alpha$, for ${ }^{8} \mathrm{Be},{ }^{12} \mathrm{C}$ and ${ }^{16} \mathrm{O}$, computed with the linked diagrams only, for the $\mathrm{B} 1$ (upper plots) and MS3 (lower plots) interactions. The upper and lower lines in each plot correspond to TICC2/1 and TICC2/2 results, respectively. The dashed line in the ${ }^{8} \mathrm{Be}$ graphs shows the effect of inclusion of the unlinked diagrams.

Figure 6 shows the variation of the ground-state energy with respect to the value of the harmonic oscillator inverse length parameter, $\alpha$. For ${ }^{8} \mathrm{Be}$, including unlinked diagrams, the minimum energy value for the $\mathrm{B} 1$ potential is $52.13 \mathrm{MeV}$, obtained with $\alpha=0.49 \mathrm{fm}^{-1}$. In the MS3 case, the optimal value is $29.03 \mathrm{MeV}$ for $\alpha=0.52 \mathrm{fm}^{-1}$. If we do not consider the unlinked diagrams we obtain very similar results, $52.30 \mathrm{MeV}$ for $\alpha=0.48 \mathrm{fm}^{-1}$ with B1 and $29.01 \mathrm{MeV}$ for $\alpha=0.52 \mathrm{fm}^{-1}$ with MS3. These results show that there is a significant shift in the values of $\alpha$ that optimize the quadratic TICC $2 / 2$ calculation from those that optimize the linear TICC2/1 ones, and an important increase in the binding energies. However, for the heavier nuclei the situation is different and the values of $\alpha$ that minimize the TICC2/1 energies lie very close to those that minimize their TICC $2 / 2$ counterparts. For ${ }^{12} \mathrm{C}$ we find that the best TICC $2 / 2$ values for the binding energy are $86.20 \mathrm{MeV}$ for $\alpha=0.58 \mathrm{fm}^{-1}$ with B1 and $50.65 \mathrm{MeV}$ for $\alpha=0.57 \mathrm{fm}^{-1}$ with $\mathrm{MS} 3$, while the optimal values found for the ${ }^{16} \mathrm{O}$ nuclei are $149.52 \mathrm{MeV}$ for $\alpha=0.62 \mathrm{fm}^{-1}$ with $\mathrm{B} 1$ and $94.86 \mathrm{MeV}$ for $\alpha=0.61 \mathrm{fm}^{-1}$ with MS3.

Since we are using a harmonic oscillator basis the size of the nucleus is mainly given by the value of the harmonic oscillator parameter, with the correlations giving a relatively small correction to this value. Once we have computed the ground-state energy (by getting the coefficients in our Gaussian expansions), we can use the obtained wavefunction to 
Table 4. Root-mean-square radii values ( $\mathrm{fm}$ ) for the nuclei and interactions considered. TICC $2 / 1^{*}$ and TICC $2 / 2^{*}$ stand for linear and linear-plus-quadratic calculations without considering unlinked diagrams. The values of the HO parameter are those given in table 2 for the TICC $2 / 1^{*}$ calculation, and the optimal ones found quoted in the text for the TICC $2 / 2^{*}$ case.

\begin{tabular}{llcccc}
\hline & & ${ }^{4} \mathrm{He}$ & ${ }^{8} \mathrm{Be}$ & ${ }^{12} \mathrm{C}$ & ${ }^{16} \mathrm{O}$ \\
\hline B1 & TICC2/1* & 1.42 & 2.25 & 2.39 & 2.42 \\
& TICC2/2* & 1.42 & 2.45 & 2.40 & 2.36 \\
MS3 & TICC2/1* & 1.46 & 2.31 & 2.42 & 2.45 \\
& TICC2/2* & 1.46 & 2.48 & 2.46 & 2.38 \\
\hline
\end{tabular}

determine the root-mean-square radius of different nuclei. This is performed by calculating the expectation value of the operator,

$$
\left\langle r^{2}\right\rangle=\frac{1}{A} \sum_{i}\left(\vec{r}_{i}-\vec{R}\right)^{2}=\frac{1}{A} \sum_{i} r_{i}^{2}-R^{2},
$$

where $\vec{R}$ is the position on the centre of mass of the system. This computation involves up to four correlation functions, since the wavefunction is quadratic, and to make it manageable we have performed it using only the linked contributions. In table 4 we show the values of the radii obtained for the nuclei and interactions studied, both for the linear and quadratic wavefunctions. The radii for the quadratic case are computed using the best values found for the $\mathrm{HO}$ oscillator parameter, and in the linear case the values of $\alpha$ are those shown in table 2. The effect of the quadratic correlations is to increase the radii of the ${ }^{8} \mathrm{Be}$ and ${ }^{12} \mathrm{C}$ nuclei, and decrease the radius of the ${ }^{16} \mathrm{O}$ nucleus. The variation is most apparent for ${ }^{8} \mathrm{Be}$ due to the big shifts in the value of the HO parameter when going from the linear TICC2/1 to the quadratic TICC $2 / 2$ correlations, and is appreciably smaller for the other two nuclei. The TICC $2 / 2$ calculations for the ${ }^{16} \mathrm{O}$ radii were performed by neglecting all the terms with four correlations, but in the ${ }^{12} \mathrm{C}$ case the differences between the full results and those with only up to three correlations are in the third decimal place. It is worth noting that if we compute the radii using the linear approach including also the unlinked contributions, the only appreciable differences occur for ${ }^{8} \mathrm{Be}$, with radii $2.24 \mathrm{fm}$ and $2.30 \mathrm{fm}$ for B1 and MS3, respectively.

\section{Conclusions}

In this paper we have used the coordinate-space form of the translationally invariant coupled cluster method to describe closed-shell nuclei within the $0 \mathrm{p}$-shell. The linear form of the wavefunction had been used before to describe these systems, but within a variational approach, computing the expectation value of the ground-state energy. Here we have translated the traditional configuration-space CCM procedure directly into coordinate-space, obtaining equations for the ground-state energy and the correlation functions by projecting the Schrödinger equation onto the uncorrelated reference state and the excited states. Working in real space solves the traditional convergence problems associated with configuration-space calculations. We have shown the importance of the quadratic terms in the TICC 2 wavefunction, which had previously been studied for bosonic systems but not for finite nuclei beyond ${ }^{4} \mathrm{He}$. The results obtained show an appreciable increase in binding energy, around $11 \%$ for ${ }^{16} \mathrm{O}$ with the MS3 interaction. Unfortunately the computation of fermionic systems is much more involved than for bosons, and the number of diagrams to be taken into account in the diagrammatical expansions is much greater because the unlinked ones (those with disconnected 
correlation functions) are no longer zero! However, when using the quadratic truncation of the wavefunction, the contribution of these diagrams is much less important than is in the linear case, at least for the computation of the ground-state energy. Thus, in future we shall include only the linked diagrams when incorporating state-dependent correlations in the wavefunction. The inclusion of state-dependent correlation functions within the linear approach produces a big increment in the binding energy of the nuclei [13], and a similar treatment for the quadratic wavefunction seems promising due to the importance of the separate contributions to the energy. In the first instance one should include in the correlation functions statedependent terms of the V4 type, before using realistic interactions, which would require at least tensor correlations. On the other hand, exploring the TICC2 is also interesting because of the possibility of using mixed schemes inspired by the Jastrow-TICI2 approach $[14,15]$. This last method, in which a scalar Jastrow factor is complemented by a TICI 2 state-dependent part, has proved to be quite successful in describing these systems, and one could think about a Jastrow-TICC2 scheme as well. One usual problem when using Jastrow-correlated wavefunctions is associated with the non-commutativity of the state-dependent correlations, which makes the calculations unmanageable for medium and heavy nuclei. Often this problem is attacked by linearizing the state-dependent part of the wavefunction, but the exploration of the TICCM may provide some insight about the more relevant terms in the wavefunction to be 'dressed' with an operatorial part.

In the mixed scheme mentioned above, the Jastrow correlations are introduced to play the role of the higher order correlations disregarded in the TICC2 wavefunction, but another point to be addressed in the future is the role of these higher order correlations within a TICCM framework. It is clear that CCM two-body terms by themselves, although they may provide a very good approximation, cannot fully control a very strong repulsive core in the interaction, thus an implementation of a TICC3 scheme which would include three-body correlations as well as two-body correlations is highly desirable. The scheme presented here can be theoretically extended to include three-body correlations in a straightforward way, but the practical implementation of this extended method may prove difficult. For the time being we are working on the extension of the current scheme to include spin and isospin correlations to match those present in the interaction, concentrating on the effect of the quadratic statedependent correlations. Once these correlations are included, the next step would be the inclusion of tensor correlations in order to deal with more realistic interactions.

\section{Acknowledgments}

This work was supported by research grants (GR/L22331 and GR/N15672) from the Engineering and Physical Sciences Research Council (EPSRC) of Great Britain. IM acknowledges useful discussions with R Guardiola and J Navarro.

\section{References}

[1] Pieper S C, Wiringa R B and Pandharipande V R 1992 Phys. Rev. C 461741

[2] Wiringa R B, Pieper S C, Carlson J and Pandharipande V R 2000 Phys. Rev. C 62014001

[3] Glöcke W and Kamada H 1993 Phys. Rev. Lett. 71971

[4] Kievsky A, Viviani M and Rosati S 1993 Nucl. Phys. A 551241

[5] Co’ G, Fabrocini A, Fantoni S and Lagaris I E 1992 Nucl. Phys. A 549439 Co’ G, Fabrocini A and Fantoni S 1994 Nucl. Phys. A 56873

[6] Arias de Saavedra F, Co' G, Fabrocini A and Fantoni S 1996 Nucl. Phys. A 605359

[7] Coester F 1958 Nucl. Phys. 7421

Coester F and Kümmel H 1960 Nucl. Phys. 17477 
[8] Kümmel H, Lührmann K H and Zabolitzky J G 1978 Phys. Rep. 36C 1

[9] Bishop R F 1991 Theor. Chim. Acta 8095

[10] Bishop R F 1998 Microscopic Quantum Many-Body Theories and Their Applications ed J Navarro and A Polls (Berlin: Springer) p 1

[11] Heisenberg J H and Mihaila B 1999 Phys. Rev. C 591440

[12] Bishop R F, Flynn M F, Boscá M C, Buendía E and Guardiola R 1990 Phys. Rev. C 421341

[13] Guardiola R, Moliner P I, Navarro J, Bishop R F, Puente A and Walet N R 1996 Nucl. Phys. A 609218

[14] Bishop R F, Guardiola R, Moliner I, Navarro J, Portesi M, Puente A and Walet N R 1998 Nucl. Phys. A 643243

[15] Buendía E, Gálvez F J, Praena J and Sarsa A 2000 J. Phys. G: Nucl. Part. Phys. 261795

[16] Guardiola R, Moliner I, Navarro J and Portesi M 1998 Nucl. Phys. A 628187

[17] Moliner I, Bishop R F, Walet N R, Guardiola R, Navarro J and Portesi M 2000 Phys. Lett. B 48061

[18] Wiringa R B, Stoks V G J and Schiavilla R 1995 Phys. Rev. C 5138

[19] Guardiola R, Moliner I, Navarro J and Portesi M 1998 Many-Body Theory of Correlated Fermion Systems ed J M Arias, M I Gallardo and M Lozano (Singapore: World Scientific) p 25

[20] Navarro J, Guardiola R, Portesi M and Moliner I 2002 Microscopic Approaches to the Structure of Light Nuclei ed R F Bishop and N R Walet (Singapore: World Scientific) at press

[21] Owen J C 1979 Ann. Phys., NY 118373

[22] Rheinboldt W C and Burkardt J 1983 ACM TOMS 9236

[23] Brink D M and Boeker E 1967 Nucl. Phys. A 911

[24] Guardiola R, Faessler A, Müther H and Polls A 1981 Nucl. Phys. A 37179

[25] Afnan I R and Tang Y C 1968 Phys. Rev. 1751337 